\title{
THE OPTIMALITY OF JAMES'S DISTORTION THEOREMS
}

\author{
P. N. DOWLING, W. B. JOHNSON, C. J. LENNARD, AND B. TURETT
}

(Communicated by Dale Alspach)

\begin{abstract}
A renorming of $\ell_{1}$, explored here in detail, shows that the copies of $\ell_{1}$ produced in the proof of the Kadec-Pełczyński theorem inside nonreflexive subspaces of $L_{1}[0,1]$ cannot be produced inside general nonreflexive spaces that contain copies of $\ell_{1}$. Put differently, James's distortion theorem producing oneplus-epsilon-isomorphic copies of $\ell_{1}$ inside any isomorphic copy of $\ell_{1}$ is, in a certain sense, optimal. A similar renorming of $c_{0}$ shows that James's distortion theorem for $c_{0}$ is likewise optimal.
\end{abstract}

James's distortion theorems for $\ell_{1}$, the space of absolutely summable sequences of scalars, and $c_{0}$, the space of null sequences of scalars, are well-known [J]. The former states that, whenever a Banach space contains a subspace isomorphic to $\ell_{1}$, the Banach space contains subspaces that are almost isometric to $\ell_{1}$. Several of the authors of this article, individually and in concert, have tried to use this feature of $\ell_{1}$ to determine if all (equivalent) renormings of $\ell_{1}$ fail to have the fixed point property for nonexpansive mappings (the FPP); i.e. if, in any renorming of $\ell_{1}$, there exist a nonempty, closed, bounded and convex subset $C$ and a nonexpansive self-map $T$ of $C$ without a fixed point. The basis of these attempts was to use the fact that $\ell_{1}$ in its usual norm fails to have the fixed point property and, since each renorming of $\ell_{1}$ contains subspaces almost isometric to $\ell_{1}$, a perturbation of the usual example would hopefully produce a nonexpansive self-map of a nonempty, closed, bounded, convex set in any renorming of $\ell_{1}$. Similar attempts in $c_{0}$ were also made. What appeared to be needed in these attempts were strengthened versions of James's distortion theorems.

To be specific, James's theorem for $\ell_{1}$ states that if a Banach space $X$ with norm $\|\cdot\|$ contains an isomorphic copy of $\ell_{1}$, then, for each $\epsilon>0$, there exists a sequence $\left(x_{k}\right)$ in the unit sphere of $X$ such that $(1-\epsilon) \sum_{k=1}^{\infty}\left|t_{k}\right| \leq\left\|\sum_{k=1}^{\infty} t_{k} x_{k}\right\| \leq$ $\sum_{k=1}^{\infty}\left|t_{k}\right|$ for all $\left(t_{k}\right) \in \ell_{1}$. The proof of the theorem shows even more than the statement indicates. The sequence $\left(x_{k}\right)$ may be chosen to have the additional property that, if $\left(\epsilon_{n}\right)$ is a sequence of positive numbers decreasing to 0 , then for each $n,\left(1-\epsilon_{n}\right) \sum_{k=n}^{\infty}\left|t_{k}\right| \leq\left\|\sum_{k=n}^{\infty} t_{k} x_{k}\right\| \leq \sum_{k=n}^{\infty}\left|t_{k}\right|$, for all $\left(t_{k}\right) \in \ell_{1}$. That is, for each $\delta>0$, by ignoring a finite number of terms at the beginning of the sequence $\left(x_{k}\right)$, one obtains copies of $\ell_{1}$ which are $(1+\delta)$-isomorphic to $\ell_{1}$. This

Received by the editors May 8, 1995 and, in revised form, July 7, 1995.

1991 Mathematics Subject Classification. Primary 46B03, 46B20.

Key words and phrases. $\ell_{1}, c_{0}$, renorming, James's distortion theorem, asymptotically isometric copies of $\ell_{1}$, fixed point property.

The second author was supported by NSF 93-06376.

The third author was partially supported by a University of Pittsburgh FAS grant.

(c)1997 American Mathematical Society 
leads one to ask if James's distortion theorem can be strengthened in the following sense:

Question. If $X$ is a Banach space that contains an isomorphic copy of $\ell_{1}$ and $\left(\epsilon_{n}\right)$ is a sequence of positive numbers that decreases to 0 , does there exist a sequence $\left(x_{k}\right)$ in the unit sphere of $X$ such that $\sum_{k=1}^{\infty}\left(1-\epsilon_{k}\right)\left|t_{k}\right| \leq\left\|\sum_{k=1}^{\infty} t_{k} x_{k}\right\| \leq \sum_{k=1}^{\infty}\left|t_{k}\right|$ for all $\left(t_{k}\right) \in \ell_{1}$ ?

The closed linear span of such a sequence $\left(x_{n}\right)$ in the above question is called an asymptotically isometric copy of $\ell_{1}$. As noted in [DL], the proof of the KadecPełczyński theorem [KP] shows that nonreflexive subspaces of $L_{1}[0,1]$ contain such "good" copies of $\ell_{1}$ and, in this case, there exist nonexpansive self-maps on closed, bounded and convex sets without fixed points. (This provides a converse to a theorem of Maurey $[\mathrm{M}]$ that every reflexive subspace of $L_{1}[0,1]$ has the FPP.) Thus, if every renorming of $\ell_{1}$ were to contain an asymptotically isometric copy of $\ell_{1}$, then every renorming of $\ell_{1}$ would fail the fixed point property. One purpose of this article is to present a renorming of $\ell_{1}$ which contains no asymptotically isometric copy of $\ell_{1}$. Thus James's distortion theorem for $\ell_{1}$ is, in this sense, optimal and the question of whether $\ell_{1}$ can be given an equivalent norm with the fixed point property remains open. Using the predual of this renorming of $\ell_{1}$, it will be seen that James's distortion theorem for $c_{0}$ is similarly optimal and the question as to whether $c_{0}$ can be given an equivalent norm with the fixed point property likewise remains open.

Recent papers ([CDL, DLT]) have extended the classes of spaces known to contain asymptotically isometric copies of $\ell_{1}$. In a related paper, Smyth [S] showed that the dual of every space $C(\Omega)$, where $\Omega$ is an infinite compact Hausdorff space, fails the weak-star fixed point property with an affine contraction.

In the ensuing discussion, $\mathbb{K}$ will denote the scalar field (the real or the complex numbers) and $\mathbb{N}$ will denote the positive integers. The Banach space $\ell_{1}$ is as usual the space of absolutely summable scalar sequences with its usual norm $\|x\|_{1}:=$ $\sum_{n=1}^{\infty}\left|\xi_{n}\right|$, for all $x=\left(\xi_{n}\right) \in \ell_{1}$. More generally, for $p \geq 1$, the Banach space of $p$-summable sequences of scalars is denoted by $\ell_{p}$ and is normed by $\|x\|_{p}:=$ $\left(\sum_{n=1}^{\infty}\left|\xi_{n}\right|^{p}\right)^{1 / p}$ for all $x=\left(\xi_{n}\right) \in \ell_{p}$. The sequence $\left(e_{n}\right)$ will always denote the canonical unit vector basis in $\ell_{p}$. Recall that $\|x\|_{p} \leq\|x\|_{1}$ for $p \geq 1$ and $x \in \ell_{1}$.

The space to be defined is, on the surface, quite simple. It is a countable sum of $\mathbb{K}$ 's and is akin to the classical $\ell_{p}$-spaces. There are two significant features to notice: the varying values of the exponents (similar to spaces of Nakano) and the placement of the parentheses in defining the norm. Fix a sequence $p=\left(p_{n}\right)$ of real numbers in $(1, \infty)$ converging to 1 . Then the space we wish to define is:

$$
\mathbb{K} \oplus_{p_{1}}\left(\mathbb{K} \oplus_{p_{2}}\left(\mathbb{K} \oplus_{p_{3}}\left(\mathbb{K} \oplus_{p_{4}} \ldots\right)\right)\right) .
$$

Let $X:=\mathbb{K}^{\mathbb{N}}$. For $x=\left(\xi_{n}\right) \in X$, define:

$$
\begin{gathered}
\nu_{1}(p, x):=\left|\xi_{1}\right|, \\
\nu_{2}(p, x):=\left(\left|\xi_{1}\right|^{p_{1}}+\left|\xi_{2}\right|^{p_{1}}\right)^{1 / p_{1}}, \\
\nu_{3}(p, x):=\left(\left|\xi_{1}\right|^{p_{1}}+\left(\left|\xi_{2}\right|^{p_{2}}+\left|\xi_{3}\right|^{p_{2}}\right)^{p_{1} / p_{2}}\right)^{1 / p_{1}},
\end{gathered}
$$




$$
\nu_{4}(p, x):=\left(\left|\xi_{1}\right|^{p_{1}}+\left(\left|\xi_{2}\right|^{p_{2}}+\left(\left|\xi_{3}\right|^{p_{3}}+\left|\xi_{4}\right|^{p_{3}}\right)^{p_{2} / p_{3}}\right)^{p_{1} / p_{2}}\right)^{1 / p_{1}} .
$$

To proceed further with this inductive construction, some notation is useful. Define the shift operator $S: X \rightarrow X$ by $S z:=\left(z_{2}, z_{3}, \ldots, z_{k}, \ldots\right)$ for all $z \in X$. For $p$ and $x$ as above define, for each $n \in \mathbb{N}$,

$$
\nu_{n+1}(p, x):=\left(\left|\xi_{1}\right|^{p_{1}}+\nu_{n}(S p, S x)^{p_{1}}\right)^{1 / p_{1}} .
$$

Each $\nu_{n}(p, \cdot)$ is a seminorm on $X$ and, for each $x \in X$, the sequence $\left(\nu_{n}(p, x)\right)_{n=1}^{\infty}$ increases to a limit $\nu_{p}(x)$. Clearly, for all $x \in X, \nu_{n}(p, x) \leq\left|\xi_{1}\right|+\cdots+\left|\xi_{n}\right|$ for every $n$. Thus $\nu_{p}(x) \leq\|x\|_{1}$ for each $x \in \ell_{1}$.

In seeking lower estimates for $\nu_{p}(x)$, first note that since all two-dimensional normed linear spaces are equivalent, two-dimensional $\ell_{q}^{2}$ is equivalent to $\ell_{1}^{2}$ and in fact, for $q \geq 1$,

$$
\left\|\left(\xi_{1}, \xi_{2}\right)\right\|_{q} \geq 2^{-1+1 / q}\left\|\left(\xi_{1}, \xi_{2}\right)\right\|_{1} .
$$

Then, with $K_{j}=2^{-1+1 / p_{j}}$,

$$
\begin{aligned}
\nu_{n}(p, x) & =\left(\left|\xi_{1}\right|^{p_{1}}+\nu_{n-1}(S p, S x)^{p_{1}}\right)^{1 / p_{1}} \\
& \geq K_{1}\left(\left|\xi_{1}\right|+\nu_{n-1}(S p, S x)\right) \\
& =K_{1}\left(\left|\xi_{1}\right|+\left(\left|\xi_{2}\right|^{p_{2}}+\nu_{n-2}\left(S^{2} p, S^{2} x\right)^{p_{2}}\right)^{1 / p_{2}}\right) \\
& \geq K_{1}\left(\left|\xi_{1}\right|+K_{2}\left(\left|\xi_{2}\right|+\nu_{n-2}\left(S^{2} p, S^{2} x\right)\right)\right) \\
& \geq K_{1} K_{2}\left(\left|\xi_{1}\right|+\left|\xi_{2}\right|+\nu_{n-2}\left(S^{2} p, S^{2} x\right)\right) \\
& \vdots \\
& \geq K_{1} K_{2} \cdots K_{n} \sum_{j=1}^{n}\left|\xi_{j}\right| .
\end{aligned}
$$

Specializing to the sequence $p=\left(p_{j}\right)$ where $p_{j}=\frac{2^{j}}{2^{j}-1}$ yields:

$$
\frac{1}{2}\|x\|_{1} \leq \nu_{p}(x) \leq\|x\|_{1} \quad \text { for all } x \in \ell_{1} .
$$

Thus, for this specific choice of $p, \nu_{p}(\cdot)$ is an equivalent norm on $\ell_{1}$.

It is clear that $\nu_{p}(\cdot)$ is equivalent to the $\ell_{1}$ norm whenever $p_{n}$ converges to 1 sufficiently quickly. Moreover, it is easy to determine for which sequences $p$ the norm $\nu_{p}(\cdot)$ is equivalent to the $\ell_{1}$ norm. The characterization is in terms of the dual norm $\nu_{q}(\cdot)$, where $q=\left(q_{1}, q_{2}, \ldots\right)$ satisfies $\frac{1}{p_{n}}+\frac{1}{q_{n}}=1$ for each $n \in \mathbb{N}$.

Proposition 1. Let $p$ be a sequence in $(1, \infty)$ and let $q$ be the sequence of conjugate exponents of $p$. Then the following are equivalent.

(i) $\nu_{p}(\cdot)$ is equivalent to the $\ell_{1}$ norm.

(ii) $\nu_{q}(\cdot)$ is equivalent to the $c_{0}$ norm.

(iii) $\lim _{n \rightarrow \infty} \nu_{q}\left(1_{[1, n]}\right)<\infty$.

(iv) There exists $\delta>0$ so that for all $n, q_{n}^{\#} \geq \delta \log n$, where $\left(q_{n}^{\#}\right)$ is the increasing rearrangement of $q$. 
Proof. We will use the notation $1_{E}$ to denote the characteristic function of a subset $E$ of $\mathbb{N}$. The equivalence of the first three conditions is well-known in a general context. That implication (iii) implies (iv) is straightforward. Indeed, since for each $n$, there are at least $n$ values of $k$ for which $q_{k} \leq q_{n}^{\#}$, we have for sufficiently large $N$ that

$$
\nu_{q}\left(1_{[1, N]}\right) \geq n^{1 / q_{n}^{\#}} .
$$

So, if we set $C:=\lim _{N \rightarrow \infty} \nu_{q}\left(1_{[1, N]}\right)$, then for all $n$,

$$
q_{n}^{\#} \geq \frac{\log n}{\log C} \text {. }
$$

For (iv) implies (iii), let $C>3^{1 / \delta}$. Then

$$
\sum_{n=1}^{\infty} C^{-q_{n}}=\sum_{n=1}^{\infty} C^{-q_{n}^{\#}}<\infty
$$

Now for $x>0, s>1,\left(1+x^{-1}\right)^{1 / s} \leq 1+s^{-1} x^{-1}$, and therefore $\left(1+x^{s}\right)^{1 / s} \leq$ $x+s^{-1} x^{1-s}$. Hence, for each $k \leq N$

$$
\nu_{q}\left(1_{[k, N]}\right) \leq \nu_{q}\left(1_{[k+1, N]}\right)+q_{k}^{-1} \nu_{q}\left(1_{[k+1, N]}\right)^{1-q_{k}} .
$$

If $\nu_{q}\left(1_{[1, N]}\right) \leq C$ for all $N \in \mathbb{N}$, then we are done. If $\nu_{q}\left(1_{[1, N]}\right)>C$, choose $m$ so that $\nu_{q}\left(1_{[m, N]}\right) \geq C>\nu_{q}\left(1_{[m+1, N]}\right)$. Then

$$
\nu_{q}\left(1_{[1, N]}\right) \leq C+1+\sum_{k=1}^{m-1} q_{k}^{-1} \nu_{q}\left(1_{[k+1, N]}\right)^{1-q_{k}} \leq C+1+C \sum_{k=1}^{\infty} q_{k}^{-1} C^{-q_{k}}<\infty .
$$

The next result shows the optimality of James's theorem by proving that the above renormings of $\ell_{1}$ fail to contain any asymptotically isometric copies of $\ell_{1}$.

Theorem 1. Let $p=\left(p_{n}\right)$ be a sequence in $(1, \infty)$, converging to 1 and such that $\nu_{p}$ is an equivalent norm on $\ell_{1}$; and let $\left(\epsilon_{n}\right)$ be a null sequence in $(0,1)$. Then there does not exist a $\nu_{p}$-normalized sequence $\left(x_{k}\right)$ in $\ell_{1}$ such that, for all $t=\left(t_{j}\right) \in \ell_{1}$,

$$
\sum_{j=1}^{\infty}\left(1-\epsilon_{j}\right)\left|t_{j}\right| \leq \nu_{p}\left(\sum_{j=1}^{\infty} t_{j} x_{j}\right) \leq \sum_{j=1}^{\infty}\left|t_{j}\right| .
$$

Proof. Without loss of generality, assume $p$ strictly decreases to 1 . In order to obtain a contradiction, assume that there exists a null sequence $\left(\epsilon_{n}\right)$ in $(0,1)$ and a $\nu_{p}$-normalized sequence $\left(x_{k}\right)$ in $\ell_{1}$ such that

$$
\sum_{j=1}^{\infty}\left(1-\epsilon_{j}\right)\left|t_{j}\right| \leq \nu_{p}\left(\sum_{j=1}^{\infty} t_{j} x_{j}\right) \leq \sum_{j=1}^{\infty}\left|t_{j}\right| \quad \text { for all } t=\left(t_{j}\right) \in \ell_{1} .
$$

By passing to a subsequence of $\left(x_{n}\right)$ if necessary, there is no loss of generality in assuming that

$$
\sum_{n=1}^{\infty} \epsilon_{n}<1
$$


Note also that there is no loss of generality in assuming additionally that the sequence $\left(x_{n}\right)$ is disjointly supported, i.e., that the support of $x_{m}$ is disjoint from the support of $x_{n}$ if $m \neq n$. Indeed this is a classical gliding hump argument. Since the closed unit ball of $\ell_{1}$ is weak-star sequentially compact with respect to the predual $c_{0}$, by passing to a subsequence, we may suppose that $\left(x_{n}\right)$ converges weak-star (and so pointwise with respect to the usual basis $\left(e_{n}\right)$ of $\ell_{1}$ ) to some $y \in \ell_{1}$. By replacing $\left(x_{n}\right)$ by the $\nu_{p}$-normalization of the sequence $\left(\frac{x_{2 j}-x_{2 j+1}}{2}\right)$, we may assume that $y=0$. As in the proof of the Bessaga-Pełczyński theorem [BP] (or see, for example [D]), by passing to a subsequence of $\left(x_{n}\right)$ which is essentially disjointly supported, truncating to obtain a disjointly supported sequence, and then normalizing, yield a block basis $\left(b_{k}\right)$ of $\left(e_{n}\right)$ which satisfies $\left(^{*}\right)$. Consequently, we henceforth assume that $\left(x_{n}\right)$ is disjointly supported.

Let $(m(k))_{k=0}^{\infty}$ be a strictly increasing sequence in $\mathbb{N} \cup\{0\}$ with $m(0)=0$ and $\left(\xi_{j}\right)_{j=1}^{\infty}$ a sequence of scalars such that, for each $k \in \mathbb{N}$,

$$
x_{k}=\sum_{j=m(k-1)+1}^{m(k)} \xi_{j} e_{j} .
$$

Let $N$ be in $\mathbb{N}$ and, in $\left({ }^{*}\right)$, set $t_{j}=1$ for $j=1, \cdots, N$ and 0 otherwise. Then, for $N \geq m(1)$ :

$$
\begin{aligned}
N-\sum_{j=1}^{N} \epsilon_{j} & \leq \nu_{p}\left(\sum_{k=1}^{N} x_{k}\right) \\
& =\left(\left|\xi_{1}\right|^{p_{1}}+\nu_{p}\left(\sum_{j=2}^{m(N)} \xi_{j} e_{j}\right)^{p_{1}}\right)^{1 / p_{1}} \\
& \leq\left(\left|\xi_{1}\right|^{p_{2}}+\nu_{p}\left(\sum_{j=2}^{m(N)} \xi_{j} e_{j}\right)^{p_{2}}\right)^{1 / p_{2}} \\
& =\left(\left|\xi_{1}\right|^{p_{2}}+\left|\xi_{2}\right|^{p_{2}}+\nu_{p}\left(\sum_{j=3}^{m(N)} \xi_{j} e_{j}\right)^{p_{2}}\right)^{1 / p_{2}} \\
& \leq\left(\left|\xi_{1}\right|^{p_{3}}+\left|\xi_{2}\right|^{p_{3}}+\nu_{p}\left(\sum_{j=3}^{m(N)} \xi_{j} e_{j}\right)^{p_{3}}\right)^{1 / p_{3}} \\
& \vdots \\
& \left.\leq\left(\left|\xi_{1}\right|^{p_{m(1)}}+\cdots+\left|\xi_{m(1)}\right|^{p_{m(1)}}+\nu_{p} \sum_{j=m(1)+1}^{m(N)} \xi_{j} e_{j}\right)^{p_{m(1)}}\right)^{1 / p_{m(1)}} \\
& \leq\left(\left\|x_{1}\right\|_{p_{m(1)}}^{p_{m(1)}}+\nu_{p}\left(\sum_{k=2}^{N} x_{k}\right)^{p_{m(1)}}\right)^{1 / p_{m(1)}} \\
& \left(\left\|x_{1}\right\|_{p_{m(1)}}^{p_{m(1)}}+(N-1)^{p_{m(1)}}\right)^{1 / p_{m(1)}} .
\end{aligned}
$$


Thus, for $N \geq m(1)$

$$
\left(N-\sum_{j=1}^{N} \epsilon_{j}\right)^{p_{m(1)}}-(N-1)^{p_{m(1)}} \leq\left\|x_{1}\right\|_{p_{m(1)}}^{p_{m(1)}} .
$$

By $\left(^{* *}\right)$, the left-hand side of the inequality tends to $\infty$ with $N$. This yields a contradiction which finishes the proof.

In the previous proof, choosing vectors of the form $x_{1}+M x_{N}$, instead of $x_{1}+$ $\cdots+x_{N}$, also leads to a contradiction (by letting $N$ and then $M$ become arbitarily large).

We note here that the proof of James's distortion theorem for $c_{0}$ gives us that if a Banach space $(X,\|\cdot\|)$ contains an isomorphic copy of $c_{0}$, then for each sequence $\left(\epsilon_{n}\right)$ of positive numbers decreasing to 0 , there exists a sequence $\left(x_{n}\right)$ in the unit sphere of $X$ such that for each $n,\left(1-\epsilon_{n}\right) \max _{k \geq n}\left|t_{k}\right| \leq\left\|\sum_{k=n}^{\infty} t_{k} x_{k}\right\| \leq\left(1+\epsilon_{n}\right) \max _{k \geq n}\left|t_{k}\right|$, for all $\left(t_{k}\right) \in c_{0}$. In order to show that James's distortion theorem for $c_{0}$ is also optimal, the construction introduced for $\ell_{1}$ can be used as long as the sequence $p=\left(p_{j}\right)$ is chosen to increase sufficiently quickly to infinity. For example, with $p_{j}=2^{j}$,

$$
\|x\|_{\infty} \leq \nu_{p}(x) \leq 2\|x\|_{\infty} \quad \text { for all } x \in c_{0} .
$$

Thus, with this choice of $p, \nu_{p}(\cdot)$ is an equivalent norm on $c_{0}$. (For other choices of $p$, we may apply Proposition 1.)

A Banach space is said to contain an asymptotically isometric copy of $c_{0}$ if, for every sequence of positive numbers $\left(\epsilon_{n}\right)$ decreasing to 0 , there exists a sequence $\left(x_{n}\right)$ in the Banach space such that $\max _{n \in F}\left(1-\epsilon_{n}\right)\left|\alpha_{n}\right| \leq\left\|\sum_{n \in F} \alpha_{n} x_{n}\right\| \leq$ $\max _{n \in F}\left(1+\epsilon_{n}\right)\left|\alpha_{n}\right|$ for all choices of scalars $\left(\alpha_{n}\right)$ and for all finite subsets $F$ of natural numbers. (Note that $\left(1+\epsilon_{n}\right)$ may be replaced by 1 in this definition.) The next result provides a useful connection between the two asymptotically isometric properties.

Theorem 2. Let $(X,\|\cdot\|)$ be a Banach space that contains an asymptotically isometric copy of $c_{0}$. Then $X^{*}$, with the dual norm, contains an asymptotically isometric copy of $\ell_{1}$.

Proof. By hypothesis, given any null sequence $\left(\epsilon_{n}\right)$ in $(0,1)$, there is a sequence $\left(x_{n}\right)$ in $X$ such that for all finite sequences of scalars $\left(\alpha_{n}\right)_{n=1}^{N}$,

$$
\max _{1 \leq n \leq N}\left(1-\epsilon_{n}\right)\left|\alpha_{n}\right| \leq\left\|\sum_{n=1}^{N} \alpha_{n} x_{n}\right\| \leq \max _{1 \leq n \leq N}\left|\alpha_{n}\right| .
$$

Let $\left(x_{n}^{*}\right)$ be a sequence of Hahn-Banach extensions to elements of $X^{*}$ of the linear functionals on the span of $\left(x_{n}\right)$ that are biorthogonal to $\left(x_{n}\right)$. Consider $x_{m}^{*}$, for some $m \in \mathbb{N}$. Then, for all vectors $x$ of the form $\sum_{n=1}^{N} \alpha_{n} x_{n}$ with $N \geq m$, we have

$$
\begin{aligned}
\left|x_{m}^{*}(x)\right| & =\left|\alpha_{m}\right|=\left(1-\epsilon_{m}\right)^{-1}\left(1-\epsilon_{m}\right)\left|\alpha_{m}\right| \\
& \leq\left(1-\epsilon_{m}\right)^{-1} \max _{1 \leq n \leq N}\left(1-\epsilon_{n}\right)\left|\alpha_{n}\right| \leq\left(1-\epsilon_{m}\right)^{-1}\|x\| ;
\end{aligned}
$$

and hence it follows that $\left\|x_{m}^{*}\right\| \leq\left(1-\epsilon_{m}\right)^{-1}$.

Set $x_{n}^{\prime}:=\left\|x_{n}^{*}\right\|^{-1} x_{n}^{*}$ for each $n \in \mathbb{N}$. Fix a sequence $\left(\alpha_{n}\right)_{n=1}^{N}$ of scalars and let $\beta_{n}=\operatorname{sign} \alpha_{n}$ for all $n$. Then, since $\left\|\sum_{n=1}^{N} \beta_{n} x_{n}\right\| \leq \max _{1 \leq n \leq N}\left|\beta_{n}\right|=1$, we have 
that

$$
\left\|\sum_{n=1}^{N} \alpha_{n} x_{n}^{\prime}\right\| \geq\left\langle\sum_{n=1}^{N} \alpha_{n} x_{n}^{\prime}, \sum_{k=1}^{N} \beta_{k} x_{k}\right\rangle=\sum_{n=1}^{N}|| x_{n}^{*} \|^{-1}\left|\alpha_{n}\right| \geq \sum_{n=1}^{N}\left(1-\epsilon_{n}\right)\left|\alpha_{n}\right| .
$$

Thus, $X^{*}$ contains an asymptotically isometric copy of $\ell_{1}$.

Combining Theorems 1 and 2, we immediately get that not every renorming of $c_{0}$ contains an asymptotically isometric copy of $c_{0}$.

Theorem 3. Let $q=\left(q_{n}\right)$ be a sequence in $(1, \infty)$, diverging to $\infty$ and such that $\nu_{q}$ is an equivalent norm on $c_{0}$; and let $\left(\epsilon_{n}\right)$ be a null sequence in $(0,1)$. Then there does not exist a sequence $\left(x_{k}\right)$ in $c_{0}$ such that, for all $\alpha=\left(\alpha_{j}\right) \in c_{0}$,

$$
\max _{n \in F}\left(1-\epsilon_{n}\right)\left|\alpha_{n}\right| \leq \nu_{q}\left(\sum_{n \in F} \alpha_{n} x_{n}\right) \leq \max _{n \in F}\left(1+\epsilon_{n}\right)\left|\alpha_{n}\right|
$$

for all finite subsets $F$ of natural numbers.

Proof. It is enough to apply Theorems 1 and 2 , after noting that the dual of $\left(c_{0}, \nu_{q}\right)$ is $\left(\ell_{1}, \nu_{p}\right)$, where $p$ is the sequence of conjugate exponents of $q$.

In closing, note that other renormings of $\ell_{1}$ exist that fail to contain asymptotically isometric copies of $\ell_{1}$. One such norm is:

$$
\|x\|^{\prime}:=\sup _{n \in \mathbb{N}} \gamma_{n} \sum_{k=n}^{\infty}\left|\xi_{k}\right| \text {, for all } x=\left(\xi_{n}\right) \in \ell_{1},
$$

where $\left(\gamma_{n}\right)$ is a fixed sequence in $(0,1)$ that strictly increases to 1 . The details needed to show that $\ell_{1}$ with this norm fails to contain asymptotically isometric copies of $\ell_{1}$ are similar to those given for the $\nu_{p}$-norm. (Although, when checking the analogue of the proof of Theorem 1 for the $\|\cdot\|^{\prime}$-norm, (after assuming, without loss of generality, that $\left(\epsilon_{n}\right)$ decreased to 0 sufficiently fast), we used the sequence $\left(x_{1}+N x_{N}\right)_{N}$ instead of $\left(x_{1}+\cdots+x_{N}\right)_{N}$. $)$

Whether $\ell_{1}$ endowed with either of the norms $\|\cdot\|^{\prime}$ or $\nu_{p}$ has the fixed point property is unknown. The norm $\|\cdot\|^{\prime}$, suggested to us by the referee of another paper, is interesting because of its link to the strengthening of James's distortion theorem described earlier.

Finally, let us consider $c_{0}$. It is shown in [DLT] that whenever a Banach space $(X,\|\cdot\|)$ contains an asymptotically isometric copy of $c_{0}$, it must fail the FPP. We remark that the spaces $\left(c_{0}, \nu_{q}\right)$ of Theorem 3 also fail the FPP. Indeed, without loss of generality, assume $\left(q_{n}\right)$ increases to $\infty$. Then a fixed point free $\nu_{q}$-nonexpansive map $T$ on a closed, bounded and convex set $C$ is provided by the usual $c_{0}$ example: i.e. let $C:=\left\{x=\left(\xi_{n}\right) \in c_{0}: 0 \leq \xi_{n} \leq 1\right.$ for all $\left.n\right\}$ and define $T(x):=\left(1, \xi_{1}, \xi_{2}, \xi_{3}, \ldots\right)$.

\section{REFERENCES}

[BP] C. Bessaga and A. Pełczyński, On bases and unconditional convergence of series in Banach spaces, Studia Math. 17 (1958), 151-164. MR 22:5872

[CDL] N. L. Carothers, S. J. Dilworth and C. J. Lennard, On a localization of the UKK property and the fixed point property in $L_{w, 1}$, Lecture Notes in Pure and Appl. Math., vol. 175, Dekker, New York, 1996, pp. 111-124. CMP 96:03

[D] J. Diestel, Sequences and Series in Banach Spaces, Springer-Verlag, New York, Berlin, Heidelberg, Tokyo, 1984. MR 85i:46020 
[DL] P. N. Dowling and C. J. Lennard, Every nonreflexive subspace of $L_{1}[0,1]$ fails the fixed point property, Proc. Amer. Math. Soc. (to appear). CMP 96:01

[DLT] P. N. Dowling, C. J. Lennard and B. Turett, Reflexivity and the fixed point property for nonexpansive maps, J. Math. Anal. Appl. 200 (1996), 653-662.

[J] R. C. James, Uniformly non-square Banach spaces, Ann. of Math. 80 (1964), 542-550. MR 30:4139

[KP] M. I. Kadec and A. Pełczyński, Bases, lacunary sequences and complemented subspaces in $L_{p}$, Studia Math. 21 (1962), 161-176. MR 27:2851

[LT] J. Lindenstrauss and L. Tzafriri, Classical Banach Spaces I : Sequence Spaces, SpringerVerlag, Berlin, Heidelberg, New York, 1977. MR 58:17766

$[\mathrm{M}] \quad$ B. Maurey, Points fixes des contractions de certains faiblement compacts de $L^{1}$, Seminaire d' Analyse Fonctionelle, Exposé no. VIII, École Polytechnique, Centre de Mathématiques (1980-1981). MR 83h:47041

[S] M. Smyth, Remarks on the weak star fixed point property in the dual of $C(\Omega)$, J. Math. Anal. Appl. 195 (1995), 294-306. CMP 96:01

Department of Mathematics and Statistics, Miami University, Oxford, Ohio 45056

E-mail address: pndowling@miavx1.acs.muohio.edu

Department of Mathematics, Texas A\&M University, College Station, Texas 77843

E-mail address: johnson@math.tamu.edu

Department of Mathematics and Statistics, University of Pittsburgh, Pittsburgh, Pennsylvania 15260

E-mail address: chris@lennext.math.pitt.edu

Department of Mathematical Sciences, OAKland University, Rochester, Michigan 48309

E-mail address: turett@vela.acs.oakland.edu 